\title{
Long-term evolution of sunspot magnetic fields
}

\author{
Matthew J. Penn ${ }^{1}$ and William Livingston ${ }^{1}$ \\ ${ }^{1}$ National Solar Observatory†, 950 N Cherry Av, Tucson AZ 85718 \\ email: mpenn@nso.edu
}

\begin{abstract}
Independent of the normal solar cycle, a decrease in the sunspot magnetic field strength has been observed using the Zeeman-split $1564.8 \mathrm{~nm}$ Fe I spectral line at the NSO Kitt Peak McMath-Pierce telescope. Corresponding changes in sunspot brightness and the strength of molecular absorption lines were also seen. This trend was seen to continue in observations of the first sunspots of the new solar Cycle 24, and extrapolating a linear fit to this trend would lead to only half the number of spots in Cycle 24 compared to Cycle 23, and imply virtually no sunspots in Cycle 25.

We examined synoptic observations from the NSO Kitt Peak Vacuum Telescope and initially (with 4000 spots) found a change in sunspot brightness which roughly agreed with the infrared observations. A more detailed examination (with 13,000 spots) of both spot brightness and line-of-sight magnetic flux reveals that the relationship of the sunspot magnetic fields with spot brightness and size remain constant during the solar cycle. There are only small temporal variations in the spot brightness, size, and line-of-sight flux seen in this larger sample. Because of the apparent disagreement between the two data sets, we discuss how the infrared spectral line provides a uniquely direct measurement of the magnetic fields in sunspots.
\end{abstract}

Keywords. Solar Cycle, Sunspot, FeI1564.8 nm

\section{Introduction}

Observations of the magnetic fields in sunspot umbrae have been carried out by Livingston at the National Solar Observatory's McMath-Pierce solar telescope atop Kitt Peak. These observations are made with a single-element detector and measure the intensity spectra of the $1564.8 \mathrm{~nm}$ Fe I g=3 spectral line and nearby atomic and molecular absorption lines. While these observations began in the 1990's, the focus then was only on the larger sunspots visible on the solar disk. During the last 10 years these observations have become more synoptic in that all sunspots visible on the solar disk are observed in this way, from solar pores to the largest umbrae. (In the following text we use the term "spots" to refer to both sunspots with penumbrae and pores without penumbrae.) After fitting several spectral lines in the data, Livingston has compiled a table of the magnetic field strength at the darkest spot location, the continuum brightness at that location (normalized to nearby quiet Sun brightness), and the line depth of several $\mathrm{OH}$ molecular lines in the spectral field-of-view. It is important here to note (1) that no polarimetry is done, only intensity spectra are used, (2) that the $1564.8 \mathrm{~nm}$ Fe I line is completely split (i.e. the Zeeman sigma components are shifted in wavelength more than their line widths) for the 1500 Gauss and larger magnetic fields seen in the spots, and (3) the splitting of the sigma components in the intensity spectrum measures the true magnetic field strength, not a vector component of the magnetic field.

$\dagger$ NSO is operated by AURA, Inc., under contract to the National Science Foundation 
We reported in Penn \& Livingston (2006) that a time series of this magnetic field data showed a decrease in the umbral magnetic field strength which was independent of the normal sunspot cycle. Also, the measurements revealed a threshold magnetic field strength of about 1500 Gauss, below which no dark pores formed. A linear extrapolation of the magnetic field trend suggested that the mean field strength would reach this threshold 1500 Gauss value in the year 2017. Furthermore, analysis of the umbral continuum brightness showed another linear trend, and extrapolation showed the umbral brightness would be equal to the quiet Sun brightness at about the same year. Finally, the molecular line depths showed a decreasing strength with time, and again the trend suggested that molecular absorption lines would disappear from the average sunspot umbra near 2017 .

Recent observations spanning from the solar interior to the solar corona clearly show that solar Cycle 24 has started. Below the solar surface, helioseismic observations of the torsional oscillations have shown that the subsurface flow maxima migrated to latitudes of $+/-23$ degrees in February of 2009 coinciding with the flow latitude at the onset of the magnetic activity for solar Cycle 23 (). At the solar surface, the sunspot number is rising http://sidc.oma.be/sunspot-data/. The magnetic polarity of solar magnetic active regions has switched since Cycle 23, and the hemispheres now show new cycle magnetic flux consistent with Hale's polarity law http://www.nso.edu/press/cycle24.html. In the solar chromosphere the spectral Ca K index has shown an increase ftp://ftp.nso.edu/idl/ cak_plot.gif. And in the solar corona, the radio emission from the Sun at $10.7 \mathrm{~cm}$ wavelength has begun to increase http://www.spaceweather.gc.ca/sx-6-eng.php, and the UV emission from the Sun has started to rise http://lasp.colorado.edu/lisird/sorce/sorce_ssi/ ts.html. And finally of note, the Solar Cycle 24 Prediction Panel from the Space Weather Prediction Center has recognized that the minimum after solar Cycle 23 was reached in December 2008 http://www.swpc.noaa.gov/SolarCycle/.

If Cycle 24 has started, we are in the rise phase of the cycle; but where exactly in the cycle are we located? The helioseismic observations can tell us based on the latitude of the torsional oscillation bands. This gives us a phase indicator which is independent of the cycle duration or the amplitude of the activity peak for the cycle. We can extrapolate the latitudinal drift of the torsional bands () and then compare the current position with the position in Cycle 23. This calculation tells us that June 2010 in Cycle 24 corresponds with February 1998 in Cycle 23. It is instructive to examine the monthly sunspot numbers for those two months; for February 1998 that value was 40, and for June 2010 that value was 13 http://sidc.oma.be/sunspot-data/. Including the 5 months preceding these times, we find that for a 6 month period Cycle 24 has shown only 0.37 times the number of spots seen in Cycle 23. By correcting for the phase of the solar cycles, we are now seeing far fewer sunspots than we saw in the preceding cycle; solar Cycle 24 is producing an anomalously low number of dark spots and pores.

\section{Recent observations}

Figure 1 shows the observations of sunspot and pore magnetic fields from Livingston's data set. The total magnetic field strength at the darkest location in the umbra or pore is plotted against the date of the measurement. The raw measurements are shown as crosses. There is a large distribution of magnetic field strengths in spots visible on the solar photosphere, and there seems to be a lower threshold for the formation of dark spots, either pores or umbrae. No measurements show that the total magnetic field strength is less than about 1500 Gauss in a dark spot, and presumably magnetic regions with maximal field strengths less than this value do not undergo convective collapse. In 
Figure 1 the annual bins of the measurements are shown as asterisks, and the standard deviation of the mean is shown as a vertical error bar on the asterisks.

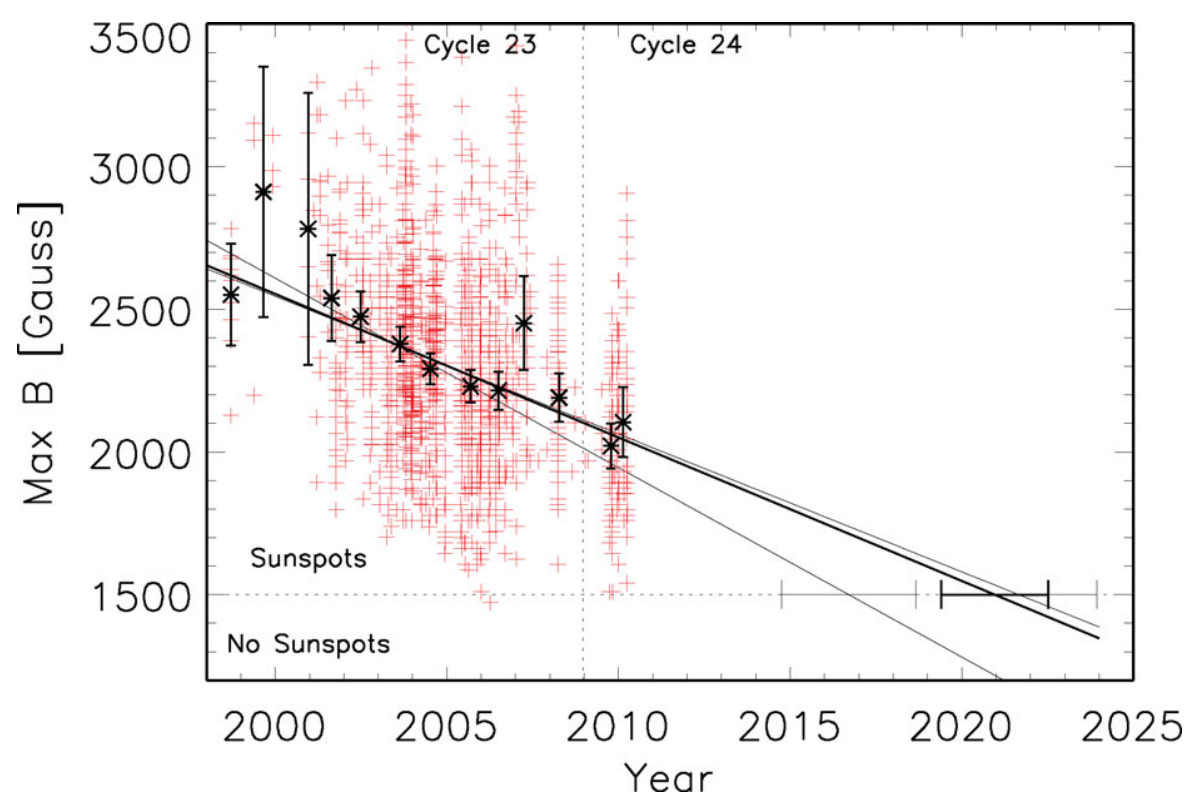

Figure 1. Measurements of the total magnetic field strength at the darkest location in umbrae and pores as a function of time. The crosses show the individual measurements, the asterisks show annual bins. Three linear fits are shown: the bottom fit line fits data from 1998-2006 as done in our 2006 paper. The top line fits all the data from Cycle 23, and the middle line fits all of the data.

Various linear fits are also shown in the Figure. The line to the left shows a linear fit from the work done by Penn \& Livingston (2006); the extrapolated line shows an intercept with the 1500 Gauss value in 2017, and error bars of the computed intercept are also shown. The right-most line fits all of the data from Livingston's Cycle 23 observations, and the slight uptick in the magnetic field measurements from 2007 and 2008 move the 1500 Gauss intercept time out to 2022. The central line fits all of the data, including measurements from Cycle 24, and the intercept date now appears to be 2021, but it is within the error-bars from the fit to the Cycle 23 sunspot data. The linear fit to all of the data show a decrease of about 50 Gauss per year in the magnetic field strength at the darkest location in spots.

It is important to note that both sunspots and pores are included in this plot. Pores, lacking penumbra, often have magnetic fields less than 2000 Gauss, but always have magnetic fields stronger than 1500 Gauss. Secondly, the intercept of the mean magnetic field strength with this 1500 Gauss threshold does not imply that all sunspots will disappear by the year 2021; rather it implies that half of the sunspots which would normally appear on the surface of the Sun would be visible. Finally, the plot doesn't address the other magnetic fields on the Sun where field strengths are lower than 1500 Gauss; the temporal behavior of solar active network or quiet Sun magnetic fields may be different from the behavior shown by sunspots. 


\section{Searching for support in other data}

The changes observed in sunspot brightness prompted an analysis of sunspot umbrae as observed in the synoptic data set from the National Solar Observatory Kitt Peak Vacuum Telescope (KPVT). In the first analysis of that data set, Penn \& MacDonald (2007) selected isolated spots by hand. About 4000 sunspots and pores were examined, and a cyclic behavior was seen in the minimum brightness found in these dark spots in phase with the sunspot cycle; darker sunspots were more common during solar maximum, and brighter sunspots were more common during solar minimum. Strangely, no significant temporal change in the radius of the sunspot umbrae was seen. Using well-known wavelength scaling coefficients from Maltby (Maltby et al. 1986) the KPVT data showed a good correspondence with both a study using MDI data (Norton \& Gilman 2004) and with the observations of sunspot intensities in the infrared by Livingston. At the time, the uptick in the magnetic field strengths seen by Livingston in 2007 and 2008 suggested that perhaps there was a solar-cycle dependence.

A more detailed analysis of the KPVT data set was performed by Tom Schad (Schad \& Penn 2010) which included an automated sunspot selection procedure, resulting in the identification of over 13,000 dark spots, and an analysis of the brightness as well as the line-of-sight magnetogram data. This work showed that there were only small temporal changes in the spot intensities and magnetic field strengths. It also showed that two empirical sunspot relationships, the first between sunspot magnetic field strength and brightness, and the second between magnetic field strength and spot radius, both remained unchanged during the solar cycle. Both relationships did contain some scatter, but it was found that the temporal changes in the spot radius were consistent with the changes in magnetic fields and brightness. Finally, current work with the KPVT data set suggests that the twist of the sunspot magnetic fields does not vary significantly during the solar cycle. The horizontal pressure balance that spots achieve with the surrounding quiet Sun behaves the same way at all phases of the solar cycle.

Work from other authors have addressed some of these issues as well. Observations of the brightness of sunspots as measured with MDI showed no changes from 1998-2004 (Mathew et al. 2007) which is consistent with the observed KPVT data during this time interval. Measurements of the brightness of sunspot umbrae from the California State University Northridge San Fernando Observatory showed no changes during the interval from 1997-2004 (Wesolowski, Walton \& Chapman 2008) although the brightness vs radius relationship from that data seems anomalous (Schad \& Penn 2010). And most recently in these proceedings, measurements of the magnetic fields from sunspot umbrae near the center of the solar disk using MDI magnetograms Watson \& Fletcher (2010) show a smaller decrease in the magnetic field strength, but that result is not significant compared to the standard errors of their fit.

Measuring the true magnetic field strength in the darkest sunspot or pore regions is known to be a difficult task since the brightness levels are low and the line depths are small (Liu, Norton \& Scherrer 2007). Using simultaneous measurements of a large sunspot from Hinode and MDI, Moon et al. (2007) show that the MDI observations can underestimate the magnetic field strength by a factor of two. Imaging magnetographs have distinct advantages in terms of cadence of observations and the spatial integrity of the images, but spectrograph-based instruments which capture full line profiles in dark spots do have advantages in terms of accuracy.

It is also important to realize that the data obtained by Livingston using the infrared line at $1564.8 \mathrm{~nm}$ with a Landé g-factor of 3.0 are measuring magnetic fields that are completely resolved. Using a conservative estimate (i.e. a large Doppler and instrumental 


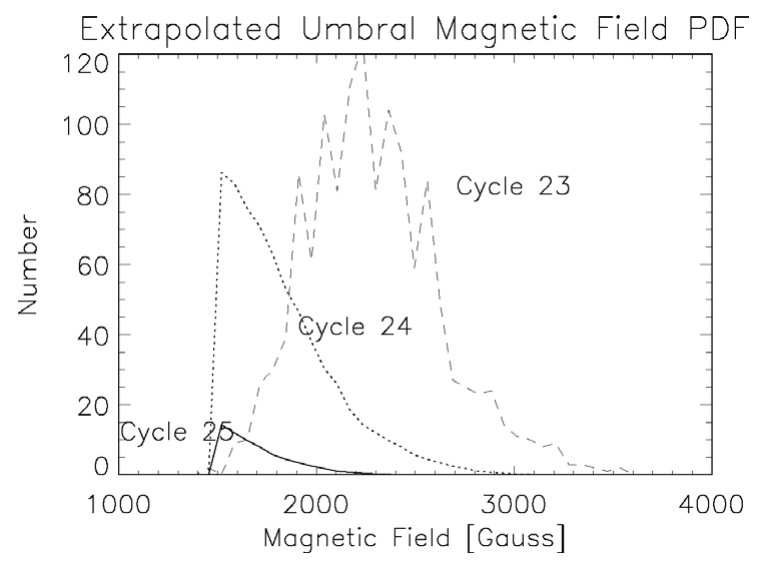

Figure 2. The magnetic probability distribution function (PDF) is show for the IR measurements of sunspots during Cycle 23. With the assumption discussed in the text, we can produce PDFs for Cycles 24 and 25. A simple scaling using the total number of spots suggests Cycle 24 will peak with a SSN of 66 , and Cycle 25 will peak with a SSN of 7 .

line width) the infrared spectral line can resolve fields with strengths greater than 750 Gauss. We can scale this value by the factor of $g$ times lambda for many of the instruments used to study sunspot magnetic fields. The KPVT magnetic field resolution would be 2400 Gauss, and for MDI the resolved field strength is about 3600 Gauss. For the HMI and SOLIS spectrograph-based instruments, the magnetic field strength must be above 2200 Gauss to be fully resolved. If we examine the measurements in Figure 1 which have magnetic fields only above 2200 Gauss, the temporal trend is not apparent. Certainly magnetic fields can be determined for spots with fields below this magnetic resolution value, but there are assumptions, corrections and (in some cases) models which are used in that determination, and perhaps the scatter inherent in that process is enough to swamp the underlying temporal variation which is so apparent in the more direct infrared measurements.

The lack of significant brightness or radius variation of sunspots as seen with other instruments is more difficult to explain. While the infrared measurements suffer from less instrumental scattered light, and perhaps better ground-based seeing than ground-based visible observations, these advantages do not seem large enough to explain the lack of variation seen with other telescopes; it remains a mystery.

\section{Implications and critical observations for the future}

As suggested by Figure 1 a detailed analysis shows that the sunspots measured during the rise phase of Cycle 24 have the same shape in the distribution of magnetic field strengths as the spots seen during the decay phase of Cycle 23, but that the mean value of the distribution is reduced. This is a conservative conclusion from Livingston's observations. If we make three assumptions however, we see that there are more dramatic implications of these infrared observations. First we assume that the distribution of magnetic fields observed by Livingston from 1998-2008 is a good proxy for the true probability distribution function (PDF) for sunspot umbral magnetic fields for Cycle 23. Secondly, we assume that the magnetic threshold of 1500 Gauss represents a real 
physical limit for the formation of a dark spot (either a pore or a sunspot) on the solar photosphere. And finally, we assume that the mean of the magnetic field PDF continues to decrease linearly with time.

Figure 2 shows the computed magnetic PDF for the sunspots in cycles 24 and 25, using a linear decrease of the magnetic field of 65 Gauss per year and a duration of 11 years for each cycle. This is meant to represent an upper limit, and the magnetic change corresponds to the most steeply sloped line in Figure 1. We can see that the PDFs for Cycle 24 and Cycle 25 vary dramatically from that observed in Cycle 23. If we assume that the appearance time of sunspots during each cycle is similar, we can use the total number of spots in each cycle to compute the maximum activity level of that cycle, using the fact that Cycle 23 showed a peak smoothed sunspot number (SSN) of 130. The linear decrease of 65 Gauss per year predicts that Cycle 24 will peak with a smoothed SSN of 66 , and Cycle 25 will peak with a smoothed SSN of 7 . Using a value of 50 Gauss per year suggests a smoothed SSN of 87 for Cycle 24 and 20 for Cycle 25.

It is important to note that it is always risky to extrapolate linear trends; but the importance of the implications from making such an assumption justify its mention. Also of note is that while these PDFs are drawn from Livingston's observations, they are at best proxies for the true sunspot magnetic PDFs. While a sunspot with a magnetic field strength of 4200 Gauss was observed in Cycle 23 (NOAA 10930, Moon et al. 2007), it was not observed by Livingston and does not appear in this analysis. Thus the sunspot which appeared recently in Cycle 24 (NOAA 11092, August 2010) with a magnetic field strength of 3350 Gauss does not invalidate these assumptions. Certainly if a large number of sunspots with magnetic field strengths greater than 3000 Gauss do appear, then the extrapolated PDF will be shown to be erroneous. We will see in the coming months and years.

Umbral magnetic field measurements at $1564.8 \mathrm{~nm}$ have been shown to reveal differences between the decay phase of Cycle 23 and the rise phase of Cycle 24, and they imply that the next two sunspot cycles might be very different from the last one. Observations with visible light magnetographs do not show significant support for these claims. Thus we feel it is essential to make synoptic observations using this very favorable infrared line to determine if these trends continue. It is essential to save the spectra and calibrations, and it would be very useful to make synoptic measurements of sunspots using temperature sensitive molecular lines such as the lines of $\mathrm{OH}$ near $1564.8 \mathrm{~nm}$.

\section{References}

Howe, R., Christensen-Dalsgaard, J., Hill, F., Komm, R., Schou, J. \& Thompson, M. J. Astrophys. J. Lett. 701, L87

Liu, Y., Norton, A. A., \& Scherrer, P. H. 2007, Solar Phys. 241, 185

Maltby, P., Avrett, E. H., Carlsson, M., Kjeldseth-Moe, O., Kurucz, R. L., \& Loeser, R. 1986, Astrophys. J. 306, 284

Mathew, S. K., Martinez Pillet, V., Solanki, S. K., \& Krivova, N. A. 2007, Astron. Astrophys 465,291

Moon, Y.-J., Kim, Y.-H., Park, Y.-D., Ichimoto, K., Sakurai, T., Chae, J., Cho, K. S., Bong, S., Suematsu, Y., Tsuneta, S., Katsukawa, Y., Shimojo, M., Shimizu, T., Shine, R. A., Tarbell, T. D., Title, A. M., Lites, B., Kubo, M., Nagata, S., \& Yokoyama, T. 2007, Pub. Astron. Soc. Jap. 59, 625 .

Norton, A. A. \& Gilman, P. A. 2004, Astrophys. J. 603, 348.

Penn, M. J. \& Livingston, W. 2006, Astrophys. J. Lett., 649, L45

Penn, M. J. \& MacDonald, R. K. D.. 2007, Astrophys. J. Lett., 662, L123 
Schad, T. A. \& Penn, M. J. 2010, Solar Phys. 262, 19

Watson, F. \& Fletcher, L. 2010, IAU Symposium 273, these proceedings

Wesolowski, M. J., Walton, S. R., \& Chapman, G. A. 2008, Solar Phys. 248, 141.

\section{Discussion}

Kosovichev: I wonder if Livingston has the data for cycle 22 to confirm your trend?

Penn: Bill's coverage in cycle 22 is really limited. So I would really like to trust his observations after 2000 .

GEORGOULIV: I was wondering if you have any additional information on how different or perhaps on how similar is the distribution of magnetic field strengths in the declining phase of the second cycle versus the rising phase of the next cycle? As we know for quite some time that the declining phases of a cycle often has surprises, that its very strong active regions. Don't forget that we had the Halloween period over the declining phase of 23. So I was wondering if there is anymore information on this apparently very complex correlation?

PenN: I must admit I have done a very bad job of looking through the literature to find out, but you beat me to some slides I didn't want to show. So here is a distribution for Cycle Number 23; and if we just move this on down using the mean decrease, here is what Bill has observed for Cycle 24 rise phase. So there is a really distinct difference between the two. So, yeah, I hope if you know of other work that has been done, I would like to see it, but it is pretty clear in his data.

OLAH: Just one comment, below 1500, what I think you have are magnetic concentrations that are not dark. So they are there. With the ASP Bruce and I were calling that azimuth centers and the typical field there would be 1400 or something, but I think it's the same that Bakers call the magnetic north. So there are magnetic structure, but they are just simply not dark. So it's a systematic effect have you there, but it's something there.

PEnN: Exactly. We're looking at the high end of the distribution of magnetic fields of the sun when we look at sunspot umbra. So yeah, either the high end - if the high end is doing something strange, then we're being misled; but maybe it's following the mean.

SAAR: Could height formation differences account for some of the differences between IR and other spot measurements?

PenN: Right. The two power laws that I showed you are mostly accounted for by the height difference. So - but in terms of the long term variation with time, I'm not sure that can be accounted by the height differences.

Strassmeier: There is full disc $\mathrm{H}$ and $\mathrm{K}$ images or K-line images that Bill had actually monitored for ages as well and do these K images show the same trend. Don't you actually have information there for, say, the global field or proxy of the global field?

Penn: Right. And from what I have seen there is no long-term trend. It's a solar cycle variation in the data set that he has. We haven't seen a long-term trend like this in that data, no. 
UnknOwn: Quick last question. Are we headed for a Maunder minimum, and is this going to solve global warming?

Penn: Well, according to the author of that book, "Red Hot Lies," I'm an outsider in solar physics; and I'm proposing this is a cause of global warming. I guess that is how I have been portrayed. So, yeah, we'll be lucky or unlucky in sunspots return, I guess. 